\title{
The Role of Powder Materials in Defense, Energy, and Security Systems
}

\author{
FERNAND D. S. MARQUIS ${ }^{1,2}$ \\ 1.-Department of Mechanical Engineering, San Diego State University, San Diego, CA 92182 , \\ USA. 2.—e-mail: fmarquis@mail.sdsu.edu
}

Multifunctional powder materials with unique combinations of properties have been synthesized recently. Among them, ultrafine materials and nanomaterials are now beginning to play a very important role in answering some of our current global challenges.

Because the properties of nanomaterials are very different from those of their conventional counterparts, they have capabilities not offered by conventional materials. This difference is so for many reasons, such as (I) dimensional changes experienced at the molecular level; (II) a consequent degree of perfection where the absence of all kinds of defects allows intrinsic properties to reach the highest values ever measured and, in certain cases, reach and surpass the theoretical limit; (III) a considerable change in specific surface area and associated properties; and (IV) the capability of development of nano-architectures not feasible in conventional materials. Among the intrinsic properties to be considered and leveraged are strength, stiffness, toughness, thermal and electrical conductivity, and others. From those associated with a specific surface are surface tension and surface concentration, catalytic and photocatalytic activity, melting point, and others. Because of these unique and exceptional properties, nanomaterials and ultrafine materials have great potential for applications in three very important 21st century industries: defense, energy, and security.

In defense, nanomaterials and ultrafine materials are now being used for many applications, such as nanoelectromechanical systems (NEMS) and microelectromechanical systems (MEMS) devices; specially engineered reactive materials; advanced penetrators; strong, tough, and lightweight vehicle

Fernand D.S. Marquis is the guest editor for the Powder Materials Committee of the TMS Materials Processing \& Manufacturing Division, and coordinator of the topic Powder Materials in Defense, Energy, and Security Systems in this issue. structures; advanced sensors; thermal protection; thermal management system; effective tank and personnel armor; and in wear and thermal protection systems.

Energy security has become a major issue as fossil fuels are confined to just a few areas in the world and their availability is controlled by political, economic, and ecological factors. A global coherent energy strategy that encompasses the entire energy life cycle is required to address all the forms of energy harvesting, storage, conversion, transmission, and distribution. In the short term, focus should be placed on achieving higher energy efficiency in all industries and processes and on increasing supplies from all forms of alternative and renewable energy, those currently available and others to be developed.

Clean water supplies have become a critical and expensive resource. Worldwide water supplies and treatment infrastructures are experiencing increasing challenges in coping with an increasing demand for clean and purified water. Currently, globally, more than 1.5 billion people do not have access to safe and adequate water supplies. Water security, like fossil fuels, is becoming a major issue, and its availability may become controlled by political, economic, and ecological factors. Here, too, there is a need to develop a global coherent water sustainability strategy that encompasses all sorts of water resources. Nanomaterials with a large active surface area and a variety of morphologies, such as nanotubes, nanorods, nanosheets, dendrimers, and nanoparticles, have the potential to impact the development of secure and cost-effective water treatment technologies.

The series of four articles assembled on this topic in this issue of $J O M$ explores the harvesting of some of these fundamental properties and their application to challenges and innovations in defense, energy, and security systems.

One of the major current challenges in the science and technology of nanomaterials is the capability of 
developing stable bulk nanomaterials capable of withstanding the working environment conditions. The article by Mark Tschopp and co-workers presents ${ }^{1}$ a review of the current state of the art and future opportunities for bulk nanomaterials with a focus on copper and copper-based alloys. The article presents and discusses the techniques and processes for the synthesis and consolidation of bulk nanomaterials and the methodology available for the stabilization of the synthesized materials at elevated temperatures. In addition, it presents and discusses the unique physical and mechanical properties of these materials and their potential applications in the defense industry.

In the processes to achieve bulk ultrafine materials and nanomaterials, consolidation and densification take a prominent role. The article by Igor Borgachev and co-workers ${ }^{2}$ presents and discusses the development of oxide-dispersion strengthened steel components by mechanical alloying and spark plasma sintering and discusses its potential applications as structural materials for nuclear reactors. Although in very recent years some significant challenges have been encountered world-wide in the safe operation of nuclear technologies, their potential as major contributors to the global energy challenge should not be dismissed, at least for now.

Still dealing with the consolidation and densification of powder materials and associated energy efficiency, the article by Ugur Cavdar and Enver Atik $^{3}$ discusses the application and the benefits of induction sintering and shows that induction sintering can be a very energy-efficient consolidation process of iron-based metal compacts.

Addressing the challenges in security and the environment, the article by Lu Pan and co-workers ${ }^{4}$ discusses the synthesis of $\mathrm{Co}_{3} \mathrm{O}_{4}$ nanomaterials with a wide range of morphologies such as nanorods, nanosheets, nanotubes, and nanoparticles and shows that they have excellent repeatability for the catalytic degradation of methyl orange and with potential applications in the remediation of polluted wastewater and soils.

Although there are still many challenges ahead in these areas, the articles presented here show that advanced powder materials and nanomaterials have the potential to play a considerable role in contributing to significant innovations in defense, energy, and security systems.

\section{REFERENCES}

1. M.A. Tschopp, K.A. Darling, H.A. Murdoch, and L. Keeskes, "Bulk" nanocrystalline metals: review of the current state of the art and future opportunities for copper and copper alloys, JOM 66 (2014). doi:10.1007/s11837-014-0978-z.

2. I. Bogachev, E. Grigoryev, O.L. Khasanov, and E. Olevsky, Fabrication of 13Cr-2Mo ferritic/martensitic oxide dispersion strengthened steel components by mechanical alloying and spark plasma sintering, JOM 66 (2014). doi:10.1007/ s11837-014-0972-5.

3. U. Cavdar and E. Atik, Investigation of conventional- and induction-sintered iron and iron-based powder metal compacts, JOM 66 (2014). doi:10.1007/s11837-014-0977-0.

4. L. Pan, L. Li, D. Tian, C. Li, and J. Wang, Synthesis of $\mathrm{Co}_{3} \mathrm{O}_{4}$ nanomaterials with different morphologies and their photo catalytic performances, JOM 656 (2014). doi:10.1007/ s11837-014-0983-2. 